\title{
Efficiency of platelet-rich plasma therapy for healing sports injuries in young athletes
}

\author{
ALEXANDRU HERDEA ${ }^{1,2}$, ALEXANDRA STRUTA $^{2}$, RĂZVAN PETRU DERIHACI $^{3}$, ALEXANDRU ULICI $^{1,2}$, \\ ADRIAN COSTACHE ${ }^{2}$, FLORENTINA FURTUNESCU ${ }^{2}$, ALEXANDRA TOMA $^{4}$ and ADHAM CHARKAOUI ${ }^{4}$ \\ ${ }^{1} 11$ th Department of Pediatric Orthopedics, 'Carol Davila' University of Medicine and Pharmacy, 050474 Bucharest; \\ ${ }^{2}$ Department of Pediatric Orthopedics, 'Grigore Alexandrescu' Children's Emergency Hospital, 011743 Bucharest, Romania; \\ ${ }^{3}$ Department of Gynecology and Obstetrics, 'Carl Gustav Carus’ University, D-01307 Dresden, Germany; \\ ${ }^{4}$ Department of Clinical Surgery, Faculty of Medicine and Pharmacy, Dunărea de Jos' University, 800008 Galați, Romania
}

Received August 6, 2021; Accepted September 7, 2021

DOI: $10.3892 /$ etm.2022.11139

\begin{abstract}
In recent years, platelet-rich plasma (PRP) therapy has been a subject of controversy in orthopedics field. Our objective was to assess the efficiency of PRP therapy for patients who have suffered grade 2 meniscal lesions and grade 2 anterior cruciate ligament (ACL) lesions, graded by magnetic resonance imaging (MRI). A retrospective observational study was conducted, which included 72 young recreational athletes who had been diagnosed with grade 2 meniscal injury, graded using MRI, that benefited from PRP therapy as an enhancement of the primary treatment, after cast immobilization. The Lysholm score, the pain intensity and the resuming of the physical activity before the PRP treatment and one month after were analyzed. Our study revealed that patients had an improved subjective perception of pain after PRP therapy and an improvement of the Lysholm score. Concurrently, $83.3 \%$ of patients could return to sports and daily physical activity. It can be concluded that PRP therapy is a safe, easy to manage treatment, efficient for pain relief and in resuming of sports activities for young recreational athletes who have sustained partial meniscal or ACL tears. In terms of pain relief, it appears that the PRP therapy could be more efficient for young patients with ACL injuries.
\end{abstract}

\section{Introduction}

The anterior cruciate ligament (ACL) and meniscus are intra-articular structures that have an inadequate healing capacity (1-4). Due to the high involvement of these structures in the knee mobility and stability $(3,4)$, injuries located at

Correspondence to: Dr Alexandru Ulici, 11th Department of Pediatric Orthopedics, 'Carol Davila' University of Medicine and Pharmacy, Bd. Eroii sanitari nr. 8, 050474 Bucharest, Romania E-mail: alexandruulici@yahoo.com

Key words: platelet-rich plasma therapy, knee, anterior cruciate ligament, meniscus, young, sports this site have always posed a challenge to the physician, this leading to various treatment protocols being developed and modified throughout the years $(5,6)$.

Biological products, such as platelet-rich plasma (PRP), mesenchymal stem cells (MSC), collagen products or hyaluronic acid, as a primary option or as an enhancement treatment, have gained increasing popularity in the previous years. New procedures have been designed and perfected to enhance the cell receptivity and, therefore, the tissue repair in various areas of the knee, such as ligaments or meniscal tissue (7-9).

Recently, teenagers and children involved in professional or recreational athletics have increased considerably, therefore generating a magnified incidence of sports-related knee trauma. Additionally, the awareness of these injuries has expanded, which results in an increased prevalence of knee lesions in the pediatric population.

PRP is an autologous blood product containing various growth factors, which have exhibited beneficial effects on cell proliferation and differentiation, extracellular matrix production, cell migration, chemotaxis and angiogenesis (10) [Table I $(11,12)]$. In addition to the multiple growth factors, desirable features of PRP include the absence of immunogenicity phenomena, emphasizing its safety levels when administered to patients $(2,8)$.

Recently, PRP has become a popular choice for soft tissue repair, for example, in aesthetic medicine (13). In terms of orthopedics and sports medicine, it has shown promising results on pain relief in both in vivo and in vitro models $(8,14-16)$.

The menisci are crescent-shaped, fibrocartilaginous structures that have a key-role in static weight-bearing, knee-stabilization, compressive force distribution, joint lubrication and proprioception (2-4). Degenerative or osteoarthritic changes emerging after total or subtotal meniscectomy emphasize the importance of preserving or repairing these structures, whenever possible, as an endeavor to maintain the normal functioning of the knee joint $(4,17)$.

When discussing the clinical outcome of meniscal injuries, the vascularization of this tissue is a significant factor. From intrauterine development and shortly after birth, the menisci are fully vascularized structures. With time, the 
vascularization subsides, thus by the age of 10 , only $10-30 \%$ of the meniscus is vascularized, and at maturity, only $10-25 \%$ of the peripheral tissue contains blood vessels $(18,19)$ In young patients, the meniscus has an adequate healing capacity, while in teenagers and children aged over 10, two models related to the location of the injury can be observed: One located in the vascular area, where the blood supply delivers nutritive factors which promote the differentiation of mesenchymal stem cells (19) and one located in the avascular area, where the healing process depends only on the intrinsic self-repair capacity of the meniscus (18-20).

According to Lotysch et al (21), on MRI, meniscal lesions can be classified into four different types: Grade 1 reveals a small focal area of hyperintensity, with no extension to the articular surface. In this case, non-operative care and physical therapy are recommended. Grade 3 abnormal meniscal signals are referred to as definite meniscal tears, and in this case, the meniscal repair is an adequate treatment (especially in athletes and active patients). Grade 2 includes linear areas of hyperintensity and no extension to the articular surface (because some grade 2 signals were found to be associated with a meniscal tear on arthroscopy, they were further subdivided into $2 \mathrm{a}, 2 \mathrm{~b}$ and $2 \mathrm{c}$ ).

Management for grade 2 meniscal lesions continue to be controversial, as numerous patients have persisting pain after conservative treatment. Blanke et al considered that this category of patients could benefit from using biological products as an enhancement of primary therapy (22).

ACL plays an important role in knee stability and normal biomechanics (6). Injuries at this site, mainly developed in sports participation, have been associated with instability, decreased physical activity, low quality of life overall and are considered to be some of the most devastating knee injuries (19). Long-term, patients with ACL lesions have the risk of developing degenerative changes in the knee, meniscal tears or chondral lesions $(23,24)$. Although reconstruction is currently the gold standard for ACL lesions, this procedure is associated with multiple complications and incomplete return to sports (25).

When addressing young patients, sports medicine and orthopedics must take into consideration the importance of ensuring that long-term complications of ACL and meniscal injuries are diminished. The clinical outcome of this age category has the singularity of a high percentage of patients involved in physical activity (recreational/professional).

Due to young patients requiring a different approach, there is a constant tendency of designing new protocols, with literature suggesting that teenagers and children who have suffered partial ACL tears, or grade 2 meniscal injuries (21), could benefit from the use of biologics, such as PRP, in terms of pain relief, joint stability improvement and resuming of physical activity $(22,26)$.

In order to comprehensively assess knee function impairment, as well as treatment efficiency, patient-reported outcome measures (PROM) are necessary.

The revised Lysholm knee scoring scale (27) consists of eight domains which evaluate: Pain (25 points), instability (25 points), locking (15 points), swelling (10 points), limp (5 points), stair climbing (10 points), squatting (5 points), and need for support (5 points). Every question response has been appointed a score on an increasing scale. The final score is represented by the sum of all the answers, and may vary from 0 to 100 . Higher scores have been associated with an improved outcome.

The Lysholm knee scoring scale has an acceptable test-retest reliability, criteria validity, construct validity, responsiveness to change (28), and it is currently used to assess various knee conditions.

\section{Materials and methods}

A retrospective observational study on 72 patients (48 females and 24 males), aged between 11 and 17 years old, was conducted on children and teenagers, defined as recreational athletes, who are involved in sports activities at school or at home, but who do not qualify as professionals. These patients were admitted to the Pediatric Orthopedics Clinic of 'Grigore Alexandrescu' Children's Emergency Hospital, (Bucharest, Romania) between January 2018 and December 2019. Inclusion criteria were as follows: patients who suffered a knee sprain and underwent $\mathrm{X}$-ray and MRI imaging, and were diagnosed with grade 2 meniscal injury and grade 2 ACL lesion. Through the use of $\mathrm{X}$-rays, the patients who had a fracture, a subchondral fracture or any other bony avulsion which would require other treatment solutions were excluded from the present study. A total of 55 patients were diagnosed with a grade 2 meniscal injury [MRI grading (21)] and 17 patients with grade 2 ACL lesion (MRI grading). They underwent PRP as an enhancement of primary treatment (after cast immobilization). The study was approved (approval no. 23223/05.12.2017) by the Ethics Committee of 'Grigore Alexandrescu' Children's Emergency Hospital (Bucharest, Romania). Preceding the study, all legal guardians of the patients signed written informed consent after receiving a detailed explanation of all the procedures the patients would undergo.

Upon admission, trauma history was recorded, and a clinical exam and X-ray were performed. After fracture cases were excluded using X-rays, the cases diagnosed as knee sprains underwent cast immobilization for three weeks. Following removal of cast immobilization, all patients followed a one-month recovery protocol, which included physiotherapy, anti-inflammatory drugs, and physical activity restraint. During this time, if there was no improvement in pain levels, joint mobility, or knee swelling, an MRI was performed. Following the MRI diagnosis, the patients underwent PRP therapy. Since it meets the criteria of a PROM, the Lysholm knee scoring scale was conducted before the first PRP injection. The patients were also requested to rate their pain intensity using the Numeric Pain Rating Scale (NPRS) (29). The same injection technique was used for all patients, and no cast immobilization was applied. All patients followed the same recovery protocol: Avoiding full weight-bearing of the injured knee for one week and gradual resumption of physical activities. For pain management, the patients had a contraindication of taking non-steroidal anti-inflammatory drugs (NSAIDs) (for six months).

A total of 1 month after the first PRP injection (3 months after the initial trauma), the clinical outcome evaluation was conducted using the Lysholm knee scoring scale and the NRS of pain. 
Table I. Growth factors and their cellular effects.

\begin{tabular}{ll}
\hline Growth factor & \multicolumn{1}{c}{ Cellular effect } \\
\hline TGF- $\beta 1$ & $\begin{array}{l}\text { Enhances the proliferative activity of } \\
\text { fibroblasts }\end{array}$ \\
FGF-2 & $\begin{array}{l}\text { Promotes angiogenesis, cell migration, cell } \\
\text { differentiation and extracellular matrix } \\
\text { production }\end{array}$ \\
IGF-1 & $\begin{array}{l}\text { Has a chemotactic effect on myoblasts } \\
\text { and fibroblasts } \\
\text { Stimulates the proliferation and chemotaxis } \\
\text { PDGF }\end{array}$ \\
VEGF & $\begin{array}{l}\text { Has a chemotactic effect on macrophages } \\
\text { and granulocytes; promotes angiogenesis }\end{array}$
\end{tabular}

TGF- $\beta 1$, transforming growth factor- $\beta 1$; FGF- 2 , fibroblast growth factor-2; IGF-1, insulin-like growth factor, PDGF, platelet-derived growth factor; VEGF, vascular endothelial growth factor.

After the first follow-up (at one month after PRP treatment), the patients included in our study had a check-up every six months.

Demographic data, pain intensity (NRS) and Lysholm score were analyzed using the unpaired t-test. The statistical significance was determined using a conventional P-value of $<0.05$ and a confidence interval (CI) of $95 \%$. Analyses were performed using Medcalc 19.4.0 software (MedCalc Software Ltd.) and Excel (Microsoft Office Pack; Microsoft Corporation).

\section{Results}

Our study included 72 subjects (48 females and 24 males), with a mean age of 14.15 vs. 14.42 years old $(\mathrm{P}=0.67)$, as demonstrated in Fig. 1. The patients were diagnosed with a grade 2 meniscal injury [MRI grading (21)] or grade 2 ACL lesion.

Pain on the NRS had a mean value of 7.81 before PRP, while at the one-month follow-up, it had a mean value of 2.22 (as revealed in Table II). The unpaired sample t-test was performed. The difference between pain before and after PRP was 5.59, with a $95 \%$ CI and an associated P-value of 0.0065 .

The Lysholm score before PRP had a mean value of 43.90 points and of 85.64 points at the one month follow up (Table II). The difference was of 41.73 points, with a $95 \% \mathrm{CI}$ and an associated P-value of 0.0004 .

In addition, the single assessment numeric evaluation of pain and the Lysholm score between the patients who had been diagnosed with ACL injuries vs. those who had been diagnosed with meniscal lesions were compared.

As demonstrated in Table III, pain levels (NRS) were similar in patients admitted with ACL lesions vs. patients admitted with meniscal lesions (7.58 vs. 7.89). The Lysholm score before the first PRP injection had a mean value of 46.11 points in patients who had sustained ACL lesions vs. 43.21 in patients who had sustained meniscus lesions (the difference was not statistically significant, $\mathrm{P}=0.63$ ).
One month after the PRP treatment, pain on the NRS decreased with a mean of 6 points in patients with ACL lesions vs. a mean decrease of 5.57 in patients with meniscal injuries. It was observed that the Lysholm score improved with a mean of 43.88 points in patients with ACL injuries, while in those who had suffered meniscal lesions, the increase had a standard of 41.07 points.

These differences between ACL and meniscal lesions, although important, were not significant statistically. It is considered that these discrepancies between the two groups could be clinically meaningful.

Regarding sports and daily physical activities, all 72 patients, before sustaining the injuries, were classified as recreational athletes. A total of 1 month after PRP treatment, 60 patients $(83.3 \%)$ were able to return to sports.

After the first one-month follow up, the patients included in our study had periodical check-ups every six months, with a mean follow-up time of 2 years. No local side effects (swelling, locking, and erythema) or worsening of symptoms were reported.

\section{Discussion}

As a primary or secondary treatment, biological products remain controversial when it comes to the field of orthopedics. Despite promising, the current literature has not yet clearly elucidated the benefits of PRP treatment for meniscal or ACL repairs.

A study conducted by Dallo et al (30) concluded that, compared with standard reconstruction procedures, biological augmentation approaches for ACL tears are associated with a faster return to sports, improved healing, and improved proprioception. This theory is in agreement with our results: An improvement in the Lysholm knee scoring scale, an adequate pain level control (NRS) and the resumption of sports from recreational athletes who have undergone PRP treatment.

In terms of meniscal healing, numerous studies (14-16) have acknowledged that PRP injections could provide an improved clinical outcome in cases where the conservative approach was unsuccessful. Although encouraging, the existing results encounter the limitations of small study groups, the biased, subjective perception of pain from patients, or lack of post-procedure MRI studies, which could support the clinical findings. Moreover, animal-based models, which depict molecular mechanisms of PRP, map a discrepancy between results (16), extending even further the controversy of the benefits of biologics for meniscal and ACL tears.

The results emerged from our study highlight the theory that there is a clear correlation between the variations in the Lysholm knee scoring scale and pain perception on the NRS [correspondingly to the conclusion reached by Sueyoshi et al (31), that although weak, this interrelationship is statistically significant], and also between the resumption of sports and daily living activities. Our findings compel us to consider that, in cases where the conservative treatment and physical therapy for partial meniscal and ACL tears fail to succeed, PRP could establish a satisfactory outcome and a significant decline of long-term complications and physical impairments.

At the one-month follow-up, none of the patients included in our study reported any local side effects or deterioration of the affected knee condition. 
Table II. Comparison of pain and Lysholm score before and after PRP.

\begin{tabular}{|c|c|c|c|c|}
\hline Variable & $\begin{array}{l}\text { Before PRP } \\
\quad \mathrm{N}=72\end{array}$ & $\begin{array}{l}\text { One month after } \\
\text { PRP N=72 }\end{array}$ & $\begin{array}{l}\text { Unpaired sample } \\
\text { t-test }\end{array}$ & $\begin{array}{l}\text { Before PRP } \\
\quad \mathrm{N}=72\end{array}$ \\
\hline $\begin{array}{l}\text { Pain }(\mathrm{NRS}), \text { mean } \\
\text { value } \pm \mathrm{SEM}, \mathrm{N}\end{array}$ & $7.819 \pm 0.1599$ & $2.22 \pm 0.2714$ & $\begin{array}{c}5.59 \\
\mathrm{P}=0.0065\end{array}$ & $7.819 \pm 0.1599$ \\
\hline $\begin{array}{l}\text { Lysholm score, mean } \\
\text { value } \pm \mathrm{SEM}, \mathrm{N}\end{array}$ & $43.90 \pm 2.244$ & $85.64 \pm 2.152$ & $\begin{array}{c}41.73 \pm 3.109 \\
P=0.0004\end{array}$ & $43.90 \pm 2.244$ \\
\hline
\end{tabular}

PRP, platelet-rich plasma; NRS, numeric rating scale; SEM, standard error of the mean.

Table III. Pain (NRS), Lysholm score variations: ACL lesions vs. meniscal lesions.

\begin{tabular}{|c|c|c|c|c|}
\hline Variable & $\begin{array}{l}\text { ACL lesions, } \\
\qquad \mathrm{N}=17\end{array}$ & $\begin{array}{l}\text { Meniscal lesions, } \\
\qquad \mathrm{N}=55\end{array}$ & $\begin{array}{l}\text { Unpaired sample } \\
\text { t-test difference }\end{array}$ & P-value \\
\hline $\begin{array}{l}\text { Pain (NRS) before PRP, } \\
\text { mean } \pm \text { SEM }\end{array}$ & $7.58 \pm 0.32$ & $7.89 \pm 0.18$ & $0.31 \pm 0.37$ & 0.63 \\
\hline $\begin{array}{l}\text { Pain }(\text { NRS) after PRP, } \\
\text { mean } \pm \text { SEM }\end{array}$ & $1.58 \pm 0.30$ & $2.41 \pm 0.36$ & $0.82 \pm 0.45$ & 0.07 \\
\hline $\begin{array}{l}\text { Pain }(\mathrm{NRS}) \text { level decrease } \\
\text { (before/after PRP) } \pm \mathrm{SEM}\end{array}$ & $\begin{array}{l}6 \pm 0.41 \\
N=17\end{array}$ & $5.57 \pm 0.36$ & $-0.52 \pm 0.54$ & 0.08 \\
\hline $\begin{array}{l}\text { Lysholm score before } \\
\text { PRP, mean } \pm \text { SEM }\end{array}$ & $46.11 \pm 4.47$ & $43.21 \pm 2.60$ & $-2.89 \pm 5.31$ & 0.09 \\
\hline $\begin{array}{l}\text { Lysholm score after } \\
\mathrm{PRP}, \text { mean } \pm \mathrm{SEM}\end{array}$ & $90 \pm 1.76$ & $84.29 \pm 2.74$ & $-5.73 \pm 3.22$ & 0.08 \\
\hline $\begin{array}{l}\text { Lysholm score increase } \\
\text { (before/after PRP), } \\
\text { mean } \pm \text { SEM }\end{array}$ & $43.88 \pm 4.76$ & $41.07 \pm 3.32$ & $-2.80 \pm 6.55$ & 0.60 \\
\hline
\end{tabular}

NRS, numeric rating scale; PRP, platelet-rich plasma; SEM, standard error of the mean; ACL, anterior cruciate ligament.

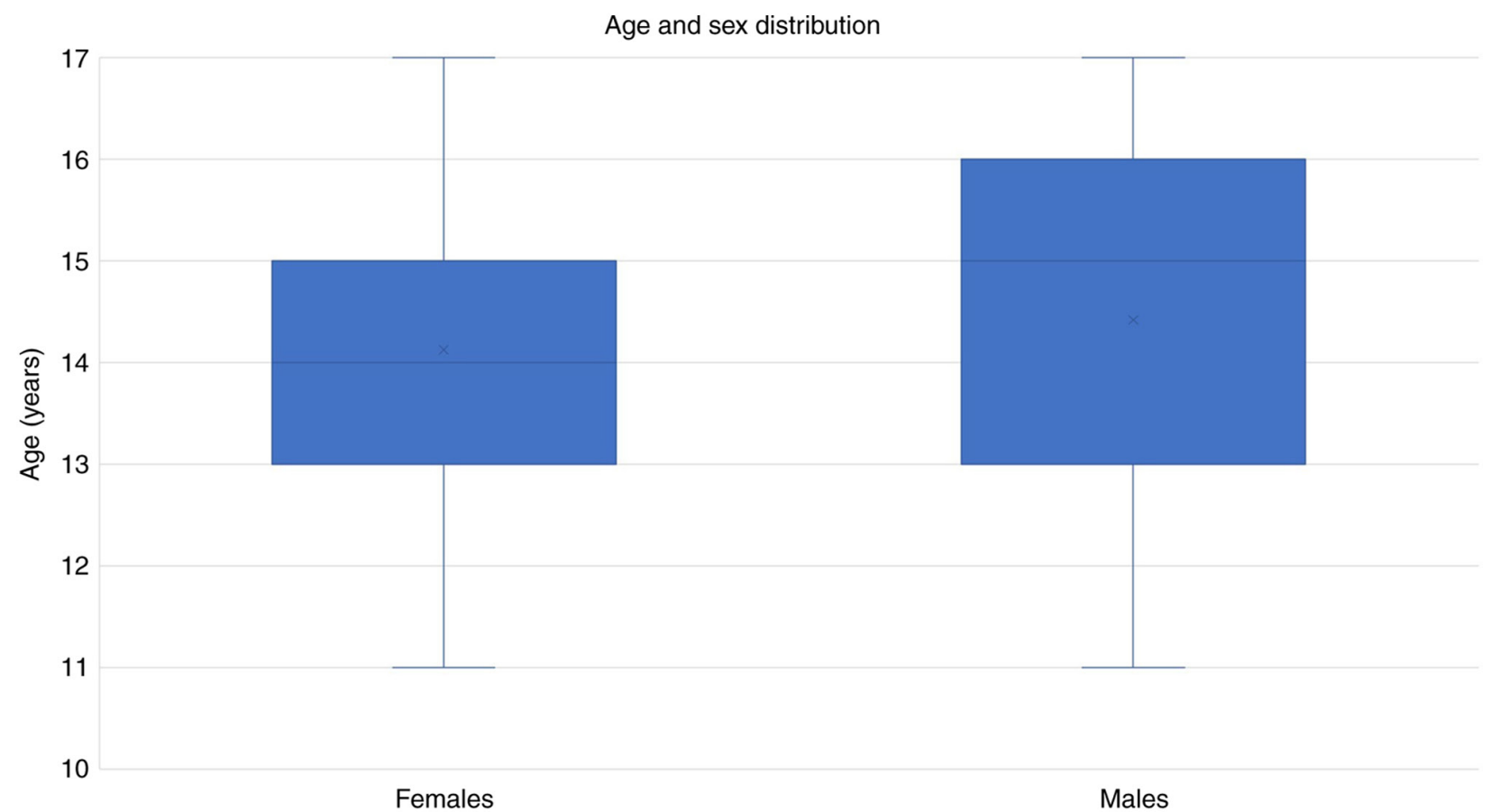

Figure 1. Patient distribution regarding age and sex. 
The main limitation of our study was the lack of MRI follow-up and the small number of cases included. Further investigation on larger groups, accompanied by post-procedure MRI, could produce an improved understanding of the role that biologics actually play in modern medicine.

In conclusion, PRP is a minimally invasive treatment, efficient for young recreational athletes, contributing to the resuming of sports at three months after the initial trauma.

Meniscal or ACL tears in adolescents can benefit from PRP in pain relief and resuming of sports.

Grade 2 ACL lesions have an improved response with PRP therapy than partial meniscal tears in terms of pain relief.

PRP is a safe, minimally invasive treatment method, without side effects, which does not require sedation or follow-up in post-intervention.

\section{Acknowledgements}

Not applicable.

\section{Funding}

No funding was received.

\section{Availability of data and materials}

All the data are registered at 'Grigore Alexandrescu' Children's Emergency Hospital, Bucharest, Romania and are available from $\mathrm{AU}$ upon reasonable request.

\section{Authors' contributions}

AH, AS, ACh and AU analyzed and interpreted the patients' data regarding the efficiency of PRP therapy in meniscal and ACL lesions, graded by MRI. AT revised the literature data and analyzed other studies where the treatment with PRP injections provided favorable clinical outcomes. RPD and ACo researched the studies that were included as references. FF designed the study, and critically revised the manuscript and approved the current form of the article in order to be submitted to the journal. All the authors read and approved the final manuscript and agree to be accountable for all aspects of the work in ensuring that questions related to the accuracy or integrity of any part of the work are appropriately investigated and resolved.

\section{Ethics approval and consent to participate}

The study was approved (approval number 23223/05.12.2017) by the Ethics Committee of 'Grigore Alexandrescu' Children's Emergency Hospital (Bucharest, Romania) which follows the World Medical Association Code of Ethics (Declaration of Helsinki, 1967). Preceding the study, all legal guardians signed a written informed consent after receiving a detailed explanation of all the procedures the patients would undergo.

\section{Patient consent for publication}

Not applicable.

\section{Competing interests}

The authors declare that they have no competing interests.

\section{References}

1. Vavken $P$ and Murray MM: The potential for primary repair of the ACL. Sports Med Arthrosc Rev 19: 44-49, 2011.

2. Seedhom BB, Dowson D and Wright V: Proceedings: Functions of the menisci. A preliminary study. Ann Rheum Dis 33: 111, 1974.

3. Renström P and Johnson RJ: Anatomy and biomechanics of the menisci. Clin Sports Med 9: 523-538, 1990.

4. Krause WR, Pope MH, Johnson RJ and Wilder DG: Mechanical changes in the knee after meniscectomy. J Bone Joint Surg Am 58: 599-604, 1976.

5. Arnoczky SP and Warren RF: Microvasculature of the human meniscus. Am J Sports Med 10: 90-95, 1982.

6. Amis AA: Anterior cruciate ligament replacement. Knee stability and the effects of implants. J Bone Joint Surg Br 71: 819-824, 1989.

7. Murray MM, Spindler KP, Ballard P, Welch TP, Zurakowski D and Nanney LB: Enhanced histologic repair in a central wound in the anterior cruciate ligament with a collagen-platelet-rich plasma scaffold. J Orthop Res 25: 1007-1017, 2007.

8. Gobbi A, Karnatzikos G, Mahajan V and Malchira S: Platelet-rich plasma treatment in symptomatic patients with knee osteoarthritis: Preliminary results in a group of active patients. Sports Health 4: 162-172, 2012.

9. Cheng M, Wang H, Yoshida R and Murray MM: Platelets and plasma proteins are both required to stimulate collagen gene expression by anterior cruciate ligament cells in three-dimensional culture. Tissue Eng Part A 16: 1479-1489, 2010.

10. Ionescu LC, Lee GC, Huang KL and Mauck RL: Growth factor supplementation improves native and engineered meniscus repair in vitro. Acta Biomater 8: 3687-3694, 2012.

11. Clendenen TV, Arslan AA, Lokshin AE, Idahl A, Hallmans G, Koenig KL, Marrangoni AM, Nolen BM, Ohlson N, Zeleniuch-Jacquotte A and Lundin E: Temporal reliability of cytokines and growth factors in EDTA plasma. BMC Res Notes 3: 302, 2010.

12. Kim ES, Kim JJ and Park EJ: Angiogenic factor-enriched platelet-rich plasma enhances in vivo bone formation around alloplastic graft material. J Adv Prosthodont 2: 7-13, 2010.

13. Samadi P, Sheykhhasan M and Khoshinani HM: The use of platelet-rich plasma in aesthetic and regenerative medicine: A comprehensive review. Aesthetic Plast Surg 43: 803-814, 2019.

14. Popescu MB, Carp M, Tevanov I, Nahoi CA, Stratila MA, Haram OM and Ulici A: Isolated meniscus tears in adolescent patients treated with platelet-rich plasma intra-articular injections: 3-Month clinical outcome. Biomed Res Int 2020: 8282460, 2020.

15. Pujol N, Salle De Chou E, Boisrenoult P and Beaufils P: Platelet-rich plasma for open meniscal repair in young patients: Any benefit? Knee Surg Sports Traumatol Arthrosc 23: 51-58, 2015.

16. Ishida K, Kuroda R, Miwa M, Tabata Y, Hokugo A, Kawamoto T, Sasaki K, Doita M and Kurosaka M: The regenerative effects of platelet-rich plasma on meniscal cells in vitro and its in vivo application with biodegradable gelatin hydrogel. Tissue Eng 13: 1103-1112, 2007.

17. Ding C, Martel-Pelletier J, Pelletier JP, Abram F, Raynauld JP, Cicuttini $\mathrm{F}$ and Jones G: Meniscal tear as an osteoarthritis risk factor in a largely non-osteoarthritic cohort: A cross-sectional study. J Rheumatolo 34: 776-784, 2007.

18. Clark CR and Ogden JA: Development of the menisci of the human knee joint. Morphological changes and their potential role in childhood meniscal injury. J Bone Joint Surg Am 65: 538-547, 1983.

19. Makris EA, Hadidi P and Athanasiou KA: The knee meniscus: Structure-function, pathophysiology, current repair techniques, and prospects for regeneration. Biomaterials 32: 7411-7431, 2011.

20. Kobayashi K, Fujimoto E, Deie M, Sumen Y, Ikuta Y and Ochi M: Regional differences in the healing potential of the meniscus-an organ culture model to eliminate the influence of microvasculature and the synovium. Knee 11: 271-278, 2004. 
21. Lotysch M, Mink J, Crues JV and Schwartz SA: Magnetic resonance imaging in the detection of meniscal injuries. Magn Reson Imaging 4: 185, 1986.

22. Blanke F, Vavken P, Haenle M, von Wehren L, Pagenstert G and Majewski M: Percutaneous injections of platelet rich plasma for treatment of intrasubstance meniscal lesions. Muscles Ligaments Tendons J 5: 162-166, 2015.

23. Lohmander LS, Englund PM, Dahl LL and Roos EM: The long-term consequence of anterior cruciate ligament and meniscus injuries: Osteoarthritis. Am J Sports Med 35: 1756-1769, 2007.

24. Lohmander LS, Ostenberg A, Englund M and Roos H: High prevalence of knee osteoarthritis, pain, and functional limitations in female soccer players twelve years after anterior cruciate ligament injury. Arthritis Rheum 50: 3145-3152, 2004.

25. Nelson IR, Chen J, Love R, Davis BR, Maletis GB and Funahashi TT: A comparison of revision and rerupture rates of ACL reconstruction between autografts and allografts in the skeletally immature. Knee Surg Sports Traumatol Arthrosc 24: 773-779, 2016.

26. Braun HJ, Wasterlain AS and Dragoo JL: The use of PRP in ligament and meniscal healing. Sports Med Arthrosc Rev 21: 206-212, 2013

27. Tegner Y and Lysholm J: Rating systems in the evaluation of knee ligament injuries. Clin Orthop Relat Res 198: 43-48, 1985.
28. Kocher MS, Steadman JR, Briggs KK, Sterett WI and Hawkins RJ: Reliability, validity, and responsiveness of the Lysholm knee scale for various chondral disorders of the knee. J Bone Joint Surg Am 86: 1139-1145, 2004.

29. Iohom G: Chapter 11-Clinical assessment of postoperative pain. In: Postoperative Pain Management: An Evidence-Based Guide to Practice. Shorten G, Carr D, Harmon D, Puig M and Browne J (eds). Saunders Elsevier, Philadelphia, PA, pp102-108, 2006.

30. Dallo I, Chahla J, Mitchell JJ, Pascual-Garrido C, Feagin JA and LaPrade RF: Biologic approaches for the treatment of partial tears of the anterior cruciate ligament: A current concepts review. Orthop J Sports Med 5: 2325967116681724, 2017.

31. Sueyoshi T, Emoto G and Yato T: Correlation between single assessment numerical evaluation score and lysholm score in primary total knee arthroplasty patients. Arthroplast Today 4: 99-102, 2017.

(6) () $\Theta$ This work is licensed under a Creative Commons International (CC BY-NC-ND 4.0) License. 